\title{
GlobeAll: Panoramic Video for an Intelligent Room
}

\author{
Mircea Nicolescu and Gérard Medioni \\ Integrated Media Systems Center \\ University of Southern California \\ Los Angeles, CA 90089-0273 \\ \{mnicoles,medioni\}@iris.usc.edu
}

\begin{abstract}
Choosing the appropriate type of video input is an important issue for any vision-based system and the right decision must take into account the specific requirements of the intended application. In the context of Intelligent Room systems, we establish several qualitative criteria to evaluate the video input component and we use them to compare three current solutions: mobile pan-tilt-zoom cameras, wide-angle lens cameras and electronic pantilt-zoom cameras. We show that electronic pan-tilt-zoom systems best satisfy our criteria.

To support this claim, we present GlobeAll, a modular four-component prototype for a vision-based Intelligent Room: a video input component that uses an electronic pan-tilt-zoom camera array, a background learning and foreground extraction component, a tracking component and an interpretation component.
\end{abstract}

\section{Introduction}

Most of the existing attempts targeted at Intelligent Environments or Perceptual User Interfaces rely on (or are limited to) visual input. Several Computer Vision techniques are involved in processing this input, typically implemented as separate modules dedicated to different tasks: background learning and foreground extraction, tracking, 3D modeling, behavior interpretation.

While general design principles and requirements for Intelligent Environments have been frequently discussed in the literature $[1,2]$, we focus here only on the problem of acquiring visual input for such systems. In this context we establish the following qualitative criteria for choosing the most appropriate type of video input:

- Region of awareness. It represents the portion of the environment being monitored, and it is given by the overall field of view.
- Region of interest. Although the system is visually aware of a larger part of the scene, it usually concentrates on a certain region, where some activity of interest is in progress. We characterize it through three criteria:

- Quality. The level of detail (resolution) of the extracted image for the region of interest must be fine enough to allow for further processing.

- Precision of location. It is desirable to know exactly where each region of interest is placed with respect to a common reference.

- Speed of redirection. The system should be able to rapidly change its region of interest. For example, in order to track a moving person, the region of interest must be moved along fast enough to avoid losing the target.

- Background model. A unique model of the background should be maintained for the entire region of awareness. Problems occur if the background model must be assembled from several (partially) overlapping parts, in the case of multiple or mobile cameras.

- Depth range. A successful system should be able to deal with both close and distant objects.

- Cost. An obvious requirement is to use affordable camera systems and supporting hardware.

There is no camera that satisfies all these criteria. In this paper, we hope to give a more systematic view on visual input for Intelligent Rooms, through a twofold contribution: In the next section, we analyze three types of camera systems and show that electronic pan-tilt-zoom cameras offer the best trade-off solution for our requirements. Then, in Section 3 we present GlobeAll, a modular prototype for a vision-based Intelligent Room, which uses an electronic pan-tilt-zoom camera array.

\section{Why electronic pan-tilt-zoom?}

Using the criteria defined above we compare three different solutions for acquiring video input in an Intelligent Room system. A summary is given in Table 1. 
Table 1. Performance of camera systems

\begin{tabular}{|c|c|c|c|c|c|c|c|}
\hline & \multirow{2}{*}{$\begin{array}{c}\text { Region of } \\
\text { Awareness }\end{array}$} & \multicolumn{3}{|c|}{ Region of Interest } & Background & Depth Range & Cost \\
\hline Mobile PTZ Camera & Small & High & Low & Slow & Bad & Good & High \\
\hline $\begin{array}{c}\text { Wide Angle Lens } \\
\text { Camera }\end{array}$ & Large & Low & High & Fast & Bad & Good & High \\
\hline $\begin{array}{c}\text { Electronic PTZ } \\
\text { Camera }\end{array}$ & Large & High & High & Fast & Good & Limited & Low \\
\hline
\end{tabular}

Mobile pan-tilt-zoom cameras. They represent a mechanical solution, where the desired region of interest is observed by mechanical movement of the camera. Potentially, such a system could observe a wide area overall, but at a given moment (and pan-tilt orientation) its visual awareness is confined to a much smaller area.

Since the entire camera resolution is dedicated for observing the region of interest, its quality is high. Not as good are the precision and speed of redirection. When receiving a pan-tilt-zoom command, the response has a certain delay until the actual movement of the camera is performed. In addition, it is never possible to know exactly how the camera is oriented. This imprecision is a major problem when trying to maintain a background model for the entire scene. The registration errors between successive frames lead to large differences between current and previous pixels, hence false positives in the detected foreground.

There is no inherent limitation in the range of depths for this type of camera system. Nevertheless, because of their mechanical components, they are quite expensive and not very robust, being subject to wear with time.

Wide-angle lens cameras. This is an optical solution, where a special lens - also known as "fish-eye lens" - is used to capture a very large field of view, thus having a good region of awareness [3]. Since this is possible only by distorting the image at its periphery, the spatial resolution varies around the optical axis. The way to use such a system is to extract a smaller region of interest and unwarp it, but this leads to a region of interest with poor quality, due to the significant loss in spatial resolution. However, the precision of location and speed of redirection are better than those for mobile pan-tilt-zoom cameras, because no mechanical movement is involved.

Although a full background model can be maintained for the whole region of awareness, the variations in spatial resolution have a major impact on its quality. Such cameras offer a good depth range, but they are an expensive solution.

Electronic pan-tilt-zoom cameras. Such a system consists of an array of inexpensive cameras mounted in a fixed setup, so that they acquire a wide overall field of view $[4,5]$. By performing an offline calibration, the camera images are registered so that a large region of awareness can be maintained as a planar mosaic.

Based on this mosaic, any intermediate view can be created from the collection of images acquired by the array of cameras. The system is functionally equivalent to a mobile camera, but it performs its pan-tilt-zoom operations electronically rather than mechanically, so it can be considered a digital solution. The region of interest has good quality, its resolution being similar to the resolution of any one camera in the array. Because the region of interest is digitally extracted, it can be redirected rapidly and precisely.

The background model can be successfully maintained over the whole region of awareness. Once the cameras have been calibrated, the position of each frame inside the mosaic never changes and is known precisely, so there are no false foreground positives due to misalignments.

In terms of depth range, the electronic pan-tilt-zoom system is slightly inferior to the other two approaches. The cameras can never be arranged so that their optical axes converge, so 3D effects are bound to occur. Typically, there is a minimum working distance of about one or two meters. Outside this range, images appear seamlessly aligned, while closer objects appear blurred. However, when used for an Intelligent Room system, the camera system is usually placed farther than this. Finally, such a system is significantly cheaper, especially when it is implemented with off-the-shelf CMOS cameras.

After evaluating these three camera systems based on the criteria previously defined, we conclude that electronic pan-tilt-zoom cameras represent the most appropriate solution for acquiring the visual input in an Intelligent Room application.

\section{Description of our system}

We developed GlobeAll, part of a wider research effort that is targeted at a modular framework for vision-based Intelligent Environments. GlobeAll is a four-component prototype that uses an electronic pan-tilt-zoom camera. The visual input is acquired by the electronic pan-tiltzoom component, which generates a planar mosaic and 
the desired arbitrary view (Virtual Camera). A background learning and foreground extraction component maintains an adaptive background model and segments moving objects as sprites. Among them, a target is selected and followed by the tracking component. Based on user-defined generic descriptions, the interpretation module analyzes the models generated by previous components (sprites, trajectories) and augments them with semantic labels. In the following sections we give a description of each of these modules. Special emphasis is put on how they were designed in order to benefit from our video input system.

\subsection{Electronic pan-tilt-zoom camera system}

We use an array of five fixed CMOS cameras, mounted on a spherical setup and oriented radially, so that they acquire a large field of view, while also maintaining some overlapping required for calibration. Essentially, we create a two-dimensional mosaic of the observed scene with geometric and photometric correction, then we generate any intermediate view, with the ability of performing electronic pan, tilt and zoom operations. Our physical camera setup, a Virtual Camera synthesized view and the planar mosaic are shown in Figure 1.

During an off-line calibration process, the images are registered by computing a full perspective transformation that aligns them to a common reference frame.

At run-time, as the input images are captured by the camera array, they are first corrected for radial lens distortion. We pre-compute pixel relocation maps, so that, in real time, we just use them as lookup tables to remap pixels and thus compensate for lens distortion [6].

In order to generate intermediate views, the images must be first registered and merged into a panorama (environment map) of the entire scene. For efficiency reasons and because our application requires less than $180^{\circ}$ pan and tilt, we chose a planar environment map (planar mosaic). Each frame is warped into this mosaic by using the perspective transformation determined in the calibration process [7].

However, just the geometric registration is not enough. Due to different light conditions, the same object may appear with different intensity from a frame to another, resulting in visible boundaries between frames in the mosaic. Therefore, we developed an intensity blending algorithm, based on the weighted average of pixel values over the transition regions between images. The intensity of the resulting pixel in the overlapping region is defined as a weighted sum of the intensities of corresponding pixels in the frames that overlap.

In order for our system to perform all the functions of a standard pan-tilt-zoom camera, we need to have the

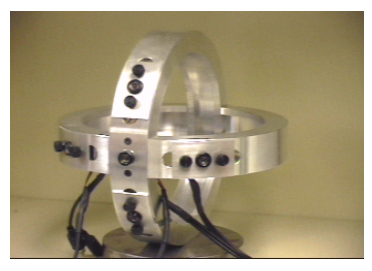

(a) Camera array

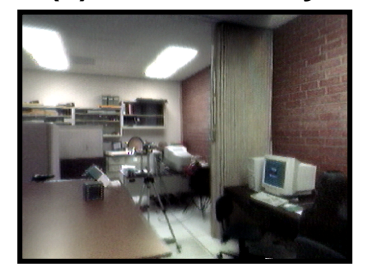

(b) Virtual Camera

\section{Figure 1. The electronic pan-tilt-zoom system.}

ability to synthesize novel views for any intermediate pantilt angles or zoom factor. What we get is a Virtual Camera, which is functionally equivalent to a regular pan-tilt-zoom platform. For this purpose, the appropriate portion of the planar mosaic is determined according to the desired pan-tilt angles and zoom factor, and then unwarped back to the novel view in the Virtual Camera.

\subsection{Background learning / foreground extraction}

The second component in our system is responsible for maintaining an adaptive (statistical) background model for the entire region of awareness, and for segmenting the moving objects that appear in foreground.

When a new frame is processed, the observed pixel values are compared to the current distribution in order to segment foreground elements. The pixels detected as foreground are then grouped in connected components, so that each moving object is represented as a sprite (silhouette and texture) [8]. After the foreground has been detected, pixel distribution values are updated in order to consider the case of a slowly changing background.

The background model is kept in mosaic space, allowing the system to detect new objects appearing anywhere in the scene, even if it happens outside the region of interest (the view of the Virtual Camera).

\subsection{Tracking}

The tracking component has two roles: to select the target from the objects detected in the previous step, and to follow the target motion, keeping it permanently within the region of interest. At this stage, the selection process is just a choice based on the size of the detected sprites.

As a target is selected, a pan/tilt command is issued for the Virtual Camera, so that it redirects the region of 

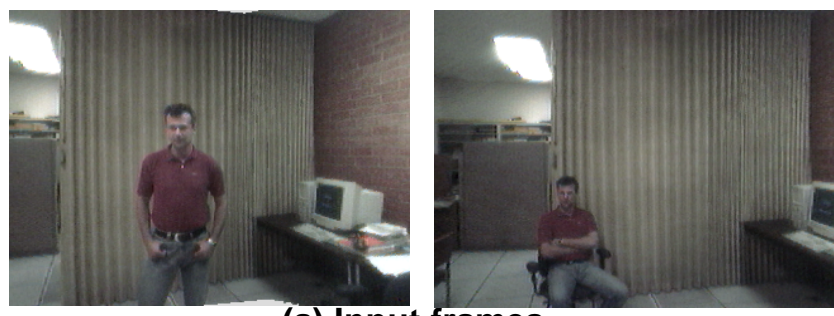

(a) Input frames
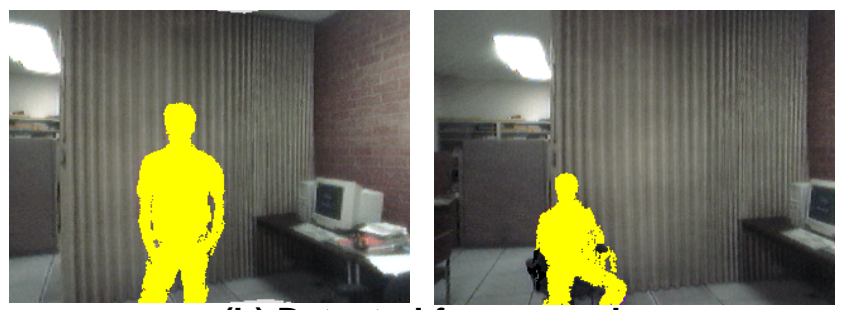

(b) Detected foreground

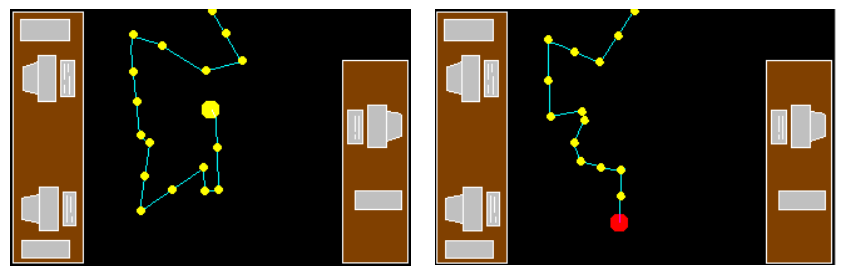

(c) Overhead trajectory and attitude

Figure 2. Foreground and 3D trajectories.

interest. The visual effect on screen is similar to that of a mobile camera tracking the moving object, although no mechanical movement is involved. The region of interest is instantly redirected, with no danger of losing the target.

\subsection{Interpretation}

From the previous modules we obtain a description of the scene in terms of planar layers (geometry and texture). These models are then augmented with semantic information by the interpretation module. Currently, this component is able to determine the $3 \mathrm{D}$ trajectories of the selected target in the room and to detect simple events such as a person standing up or sitting down on a chair.

The position of the person in the room is computed as follows: by knowing the floor position and detecting the person's head we determine the person's height and thus the head position in 3D. The trajectory is then built by tracking the head, assuming that it will be never occluded. As we retrieve the position in the room based on the person's height, a simple event such as sitting on a chair is recognized by a sudden change in location. The results are shown in Figure 2, where a yellow (light) spot represents "standing" attitude and a red (dark) spot represents "sitting" attitude.

\section{Conclusions}

In the context of Intelligent Room systems, we have studied the requirements imposed on their video input. We established six qualitative criteria and analyzed three types of camera systems as potential solutions. The electronic pan-tilt-zoom camera array is shown to perform best with respect with these criteria. It captures a large overall field of view with enough resolution, it has a better precision and response time in redirecting the region of interest and it is cheaper and more robust because it is based on a digital solution.

To support our claim, we developed GlobeAll, a fourcomponent prototype for a vision-based Intelligent Room system, using an electronic pan-tilt-zoom camera array.

\section{Acknowledgements}

We thank Dr. Mi-Suen Lee for her advice during the design of the electronic pan-tilt-zoom component. This research has been funded in part by the Integrated Media Systems Center, a National Science Foundation Engineering Research Center, Cooperative Agreement No. EEC-9529152, with additional support from the Annenberg Center for Communication at the University of Southern California and the California Trade and Commerce Agency. The support of the Philips Multimedia Center is also gratefully acknowledged.

\section{References}

[1] M. Coen, "Design Principles for Intelligent Environments", Proc. AAAI-98, Madison, WI, July 1998, pp. 547-554.

[2] R. Szeliski, "Video mosaics for virtual environments", IEEE Computer Graphics and Applications, 1996, 16(2), pp. 22-30.

[3] Interactive Pictures Corporation, http://www.ipix.com.

[4] R. Swaminathan, S. Nayar, "Polycameras: Camera Clusters for Wide Angle Imaging", Technical Report CUCS-013-99, Columbia University, April 1999.

[5] V. Nalwa, "A True Omnidirectional Viewer", Technical Report, Bell Laboratories, February 1996.

[6] H. Sawhney, R. Kumar, "True Multi-Image Alignment and its Application to Mosaicing and Lens Distortion Correction", IEEE Trans. on PAMI, 1997,.21(3), pp. 235-243.

[7] Y. Xiong, K. Turkowski, "Registration, Calibration and Blending in Creating High Quality Panoramas", Proc. WACV98, 1998, pp. 69-74.

[8] A. François, G. Medioni, "Adaptive Color Background Modeling for Real-Time Segmentation of Video Streams", Proc. of the ICISST, Las Vegas, NV, June 1999, 227-232. 